\title{
Coagulation Profile In COVID-19
}

\author{
Ambreen Kashif ${ }^{*}$, Sadia Taj ${ }^{2}$, Saira Moin ${ }^{3}$, Syeda Arzinda Fatima ${ }^{4}$, Ayaz Lone ${ }^{5}$ and Qasim \\ Ahmed ${ }^{6}$
}

${ }^{1}$ Senior Demonstrator, Fatima Memorial Hospital, Lahore, Pakistan

${ }^{2}$ Assistant professor, Fatima Memorial Hospital, Lahore, Pakistan

${ }^{3}$ Senior Demonstrator, Fatima Memorial Hospital, Lahore, Pakistan

${ }^{4}$ Assistant Professor Medicine, Fatima Memorial Hospital, Lahore, Pakistan

${ }^{5}$ Professor of Hematology, Fatima memorial Hospital, Lahore, Pakistan

${ }^{6}$ Head of Department Pathology, Fatima Memorial Hospital, Lahore, Pakistan

*Corresponding author: Ambreen Kashif, Senior Demonstrator, Fatima Memorial Hospital, Lahore, Pakistan.

To Cite This Article: Ambreen Kashif, Sadia Taj, Saira Moin, Syeda Arzinda Fatima, Ayaz Lone, Qasim Ahmed. Coagulation Profile In COVID-19. Am J Biomed Sci \& Res. 2021 - 14(1). AJBSR.MS.ID.001953. DOI: 10.34297/AJBSR.2021.14.001953.

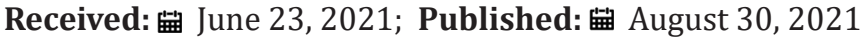

\begin{abstract}
Background: COVID-19 not only involves respiratory system but also affects other systems of the body. The diversity in symptoms is seen in affected individuals in whom some of them have mild to moderate symptoms who recover without hospitalization and the other group with critical to severe symptom need hospitalization with intensive treatment plans. The association of coagulation dysfunction with disease progression of COVID-19 is proven by much research. The main objective of our study is to evaluate the association of coagulation profile with mortality.

Materials and methods: Pathology and medicine department, FMH college of Medicine and Dentistry conducted a retrospective study. Total of 101, confirmed cases of COVID-19 disease, both genders between 17 and 75-year age were included. Coagulation profile was compared between survivor and non-survivor groups. Continuous variables were analyzed by using independent sample T-test while categorical variables were analyzed by chi-square test. Data was analyzed in SPSS version 25. p-value $\leq 0.05$ was considered significant.

Results: Out of 101 COVID-19 positive patients, 93(92.1\%) patients were in the survivor group and 08 (7.8\%) patients were in the non-survivor group. Mean APTT (p-0.02) in non-survivor group showed relatively higher values than survivor group. Similarly, Mean D-Dimers (p-0.007) in non-survivors showed a significantly raised values. However other parameters of coagulation like Platelets, MPV, PT and INR showed no significant association statistically.
\end{abstract}

Conclusion: This study infers that high D-dimers and prolonged APTT are associated with mortality in COVID-19 Patients.

Keywords: Coagulation, PT, APTT, D-Dimer, COVID-19, Non-Survivor, Infectious Respiratory, Morbidity, Novel Corona Virus, D-Dimers, Neutrophil, Widespread, Pathophysiology.

\section{Introduction}

The novel Corona virus disease is an infectious respiratory tract infection which has affected more than 166 million patients globally. Association of COVID-19 disease with other body systems is well established. COVID-19 infections are found to be associated with variable degrees of coagulopathy and severe cases show a higher incidence of COVID-19 associated coagulopathy that may increase the morbidity and mortality [1]. Thromboembolic complications have been reported in 21 to $69 \%$ of ICU patients with COVID-19 [2]. Severe COVID-19 illness is associated with robust inflammation, which leads to hypercoagulable state. The underlying pathophysiology leading to hypercoagulable state has not been clearly understood. However, ongoing research propose that the early episodes occur in the lungs which causes severe inflammatory reaction in the alveoli, along with rise in acute phase proteins including fibrinogen. Neutrophil Extracellular Traps (NETs), which are recently documented mediators of thrombosis 
also play a role in this process [4]. A dysfunctional cascade of inflammatory thrombosis is started in pulmonary vasculature as an effect of this hyperinflammatory response. This leads to a phase of local coagulopathy. A widespread hypercoagulable state develops in more sever patients which lead to thrombosis in macro and micro vasculature [5]. Development of coagulopathy is one of the most significant markers of poor prognosis in seriously sick COVID-19 patients [3] and leads to thrombotic events both in the venous and arterial circulations.

This has been linked to excessive inflammation, platelet activation, endothelial dysfunction, and stasis. Although coagulation abnormalities in severe COVID-19 mimic other systemic coagulopathies associated with severe infections, such as DIC (Disseminated intravascular coagulation) or thrombotic microangiopathy, but certain distinct features such as very high D-dimer levels and only moderately decreased platelet counts have been reported only in COVID-19 [6]. Coagulation abnormalities are associated with a higher mortality rate and derangement in various coagulation parameters have been documented in COVID 19 patients according to different reports [7]. Decreased platelet counts were found to be related with augmented risks of more severe forms of disease and more hospital mortality in SARS-CoV-2 patients [8]. Additionally, slight elongations in Prothrombin Time (PT) and Activated Partial Thromboplastin Time (APTT), and slight thrombocytopenia have been observed in some patients whereas immensely raised levels of D-Dimer are seen in several patients, that seem to have a prognostic value. The levels of D-Dimers and Fibrinogen Degradation Products (FDPs) are found to be higher in patients with severe COVID-19 infection that the patients who have milder state of disease1. This study is aimed to evaluate the coagulation profile in COVID-19 patients and to correlate disease severity with the coagulation parameters.

\section{Materials and Methods}

Pathology and Medicine Department, FMH college of Medicine and Dentistry conducted a retrospective cross-sectional study. Data of three months (MAY-JUNE 2020) was recruited after taking approval form Institutional Review Board (IRB\# FMH-08-2020-IRB776-M). Total of 101 COVID-19 PCR positive patients, both genders and age ranging between 17 and 75 years were included in this study. Patients with known hematological disorders and known history of chronic liver disease were omitted. Patients were categorized in to two groups that is survivor and non-survivor groups. Coagulation profile was performed on CA-550 and Complete Blood Count (CBC) was performed on Sysmex XN-10. Coagulation profile in survivor and non-survivor groups was statistically assessed. The platelet count was categorized as normal $\geq 150 \times 10^{3} / \mathrm{ul}$ and low $<150 \times 10^{3} /$ ul. Similarly, the PT $\geq 14$ seconds was considered prolonged PT. INR of $\geq 1.3$ was categorized as increased, APTT of $\geq 32$ seconds was considered prolonged and D-Dimers of $\geq 0.5$ was labelled as raised.

\section{Statistics}

Descriptive analysis was performed on all the variables. Data was stratified into survivor and non-survivor groups. Hematological and coagulation parameters were compared between these groups. Quantitative variables like Mean Platelet Volume (MPV), Platelet counts, Activated Partial Thromboplastin Time (APTT), Prothrombin Time (PT) and D-dimers were presented as Mean \pm SD whereas. Continuous variables were analyzed by using independent sample T-test while categorical variables were analyzed by Chisquare test. Categorical variables like gender were presented in the form of percentage and frequency. Data was analyzed in SPSS version 25 . P-value $\leq 0.05$ was considered significant.

\section{Results}

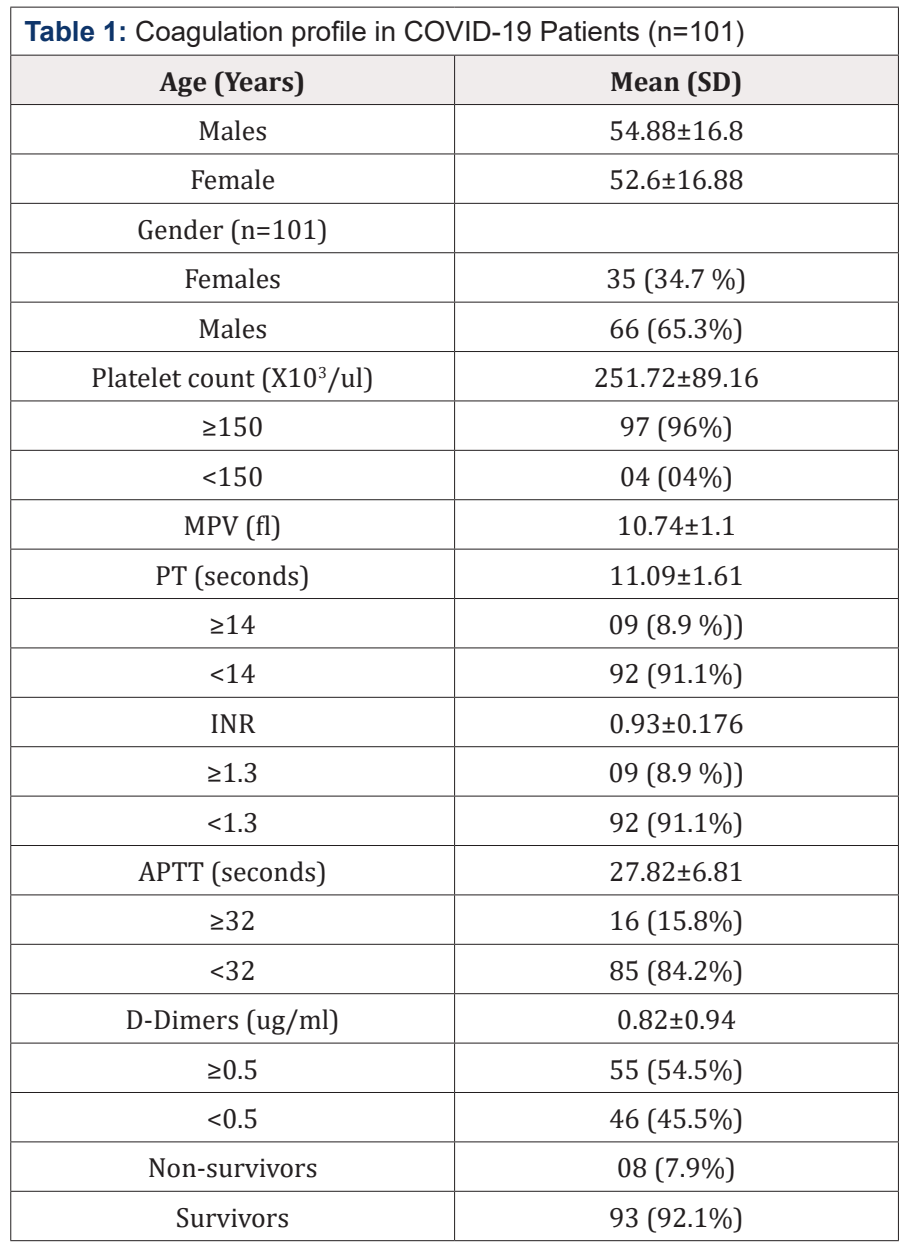

Out of 101 COVID -19 patients, there were 66(65.3\%) males and $35(34.7 \%)$ females. The Mean age of males was $54.8 \pm 16.8$ years and $52.6 \pm 16.8$ years of females. Coagulation parameters in all the COVID-19 patients showed that 97(96\%) patients had normal platelet count while 04(4\%) patients showed thrombocytopenia. 
Among 101 patients, 9(8.9\%) patients showed prolonged PT ( $\geq 14$ seconds) and INR $(\geq 1.3)$. APTT was prolonged in $16(15.8$ $\%$ ) patients and D-Dimers were raised in 55(54.5\%) of COVID-19 positive patients (Table1). The data of coagulation profile was then stratified in to two groups (survivor and non-survivor), which showed that patients in the survivor group were younger as compared to patients in non-survivor group. Mean age was $53.5 \pm 16.5$ years and $60.88 \pm 20.5$ years in survivor and nonsurvivor, respectively. Total percentage of mortality in COVID-19 patients was $08(7.8 \%)$, among them $07(87.1 \%)$ were males while $01(2.9 \%)$ was female. In coagulation profile, mean platelet count was $244 \pm 86.7 \mathrm{X} 10^{3} / \mathrm{ul}$ in survivor group while $183 \pm 94.57 \mathrm{X} 10^{3} / \mathrm{ul}$ in non-survivors ( $p$-value 0.285 ).

\begin{tabular}{|c|c|c|c|}
\hline & Survivors & Non-Survivors & P-value \\
\hline Gender (n=101) & & & 0.173 \\
\hline Male & $59(89.4 \%)$ & $07(10.7 \%)$ & \\
\hline female & $34(97.1 \%)$ & $01(2.9 \%)$ & \\
\hline Age (years) & $53.5 \pm 16.5$ & $60.88 \pm 20.5$ & 0.236 \\
\hline Platelet (X103/ul) & $257.63 \pm 86.7$ & $183.13 \pm 94.57$ & 0.285 \\
\hline$\geq 150$ & $90(96.8 \%)$ & $7(87.5 \%)$ & \\
\hline$<150$ & $03(3.2 \%)$ & $01(12.5 \%)$ & \\
\hline MPV (fl) & $10.68 \pm 0.99$ & $11.49 \pm 1.9$ & 0.05 \\
\hline PT (seconds) & $10.7 \pm 1.45$ & $12 \pm 2.62$ & 0.149 \\
\hline$\geq 14$ second & $07(7.5 \%)$ & $02(25.0 \%)$ & \\
\hline$<14$ second & $86(92.5 \%)$ & $06(75.0 \%)$ & \\
\hline INR & $0.9 \pm 0.16$ & $1.0 \pm 0.27$ & 0.149 \\
\hline$\geq 1.3$ & $07(7.5 \%)$ & $02(25.0 \%)$ & \\
\hline$<1.3$ & $86(92.5 \%)$ & $06(75.0 \%)$ & \\
\hline APTT (seconds) & $26.5 \pm 7.0$ & $29.85 \pm 3.64$ & 0.021 \\
\hline$\geq 32$ & $12(12.9 \%)$ & $04(50 \%)$ & \\
\hline$<32$ & $81(87.1 \%)$ & $04(50 \%)$ & \\
\hline D-dimers (ug/ml) & $0.81 \pm 0.98$ & $0.94 \pm 0.22$ & 0.007 \\
\hline$\geq 0.5$ & $47(50.5 \%)$ & $08(100 \%)$ & \\
\hline$<0.5$ & $46(49.5 \%)$ & $0(0 \%)$ & \\
\hline
\end{tabular}

Low platelet count among Survivor group was found in 3(3.2\%) while $90(96.8 \%)$ patients had normal platelet count. In nonsurvivor group, $1(12.5 \%)$ patient showed low platelet count. Mean, Mean Platelet Volume (MPV) was $10 \pm 0.99 \mathrm{Fl}$ in survivor group and 11.2 $\pm 1.9 \mathrm{Fl}$ in non-survivors. The Prothrombin Time (PT) and INR were also assessed in both groups and mean PT was $10.7 \pm 1.45$ seconds in survivor group, while $12.0 \pm 2.62$ seconds in non-survivor group. Mean INR was $0.9 \pm 0.16$ in survivor group while $1.0 \pm 0.27$ was in non-survivor group ( $p$-value 0.149). In survivor group, 07(7.5) patients had prolonged PT and INR while in non-survivors 02(25\%) patients had prolonged PT and INR. APTT showed mean of $26.5 \pm 7$ seconds in survivor group and $29.85 \pm 3.64$ seconds in non-survivor group. Among survivors $12(12.9 \%)$ patients has prolonged APTT while among non-survivors 04(50\%) patients had prolonged APTT ( $p-0.02)$. Mean D-Dimers was $0.81 \pm 0.98$ and $0.94 \pm 0.22$ in survivor and non-survivor group, respectively. Out of 93 patients of survivors, 47(50.5\%) had raised D-Dimer values while all the $08(100 \%)$ patients in non survivors showed elevated levels of D-Dimers ( $p$-0.007) (Table2) (Figure A-G)

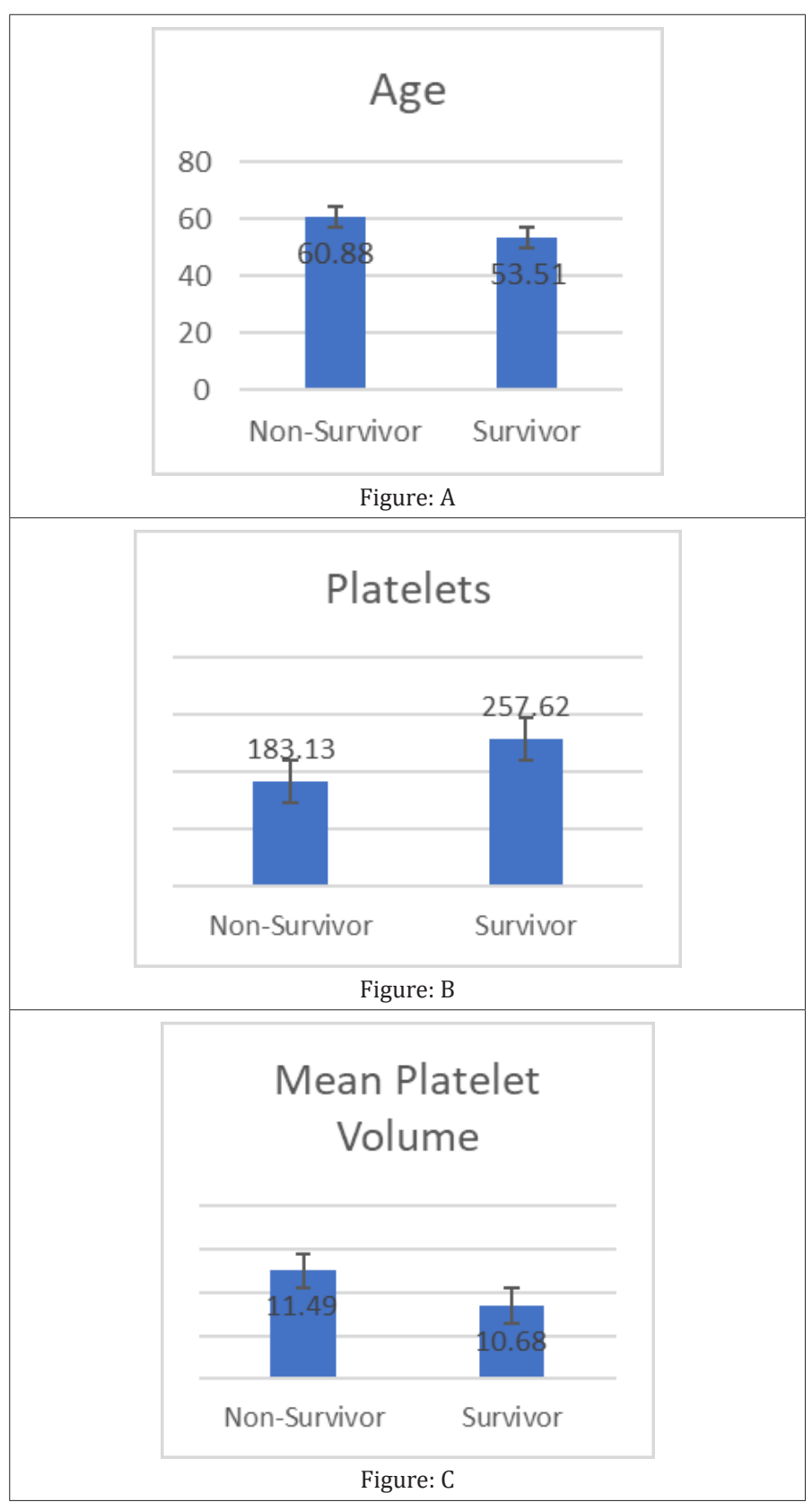




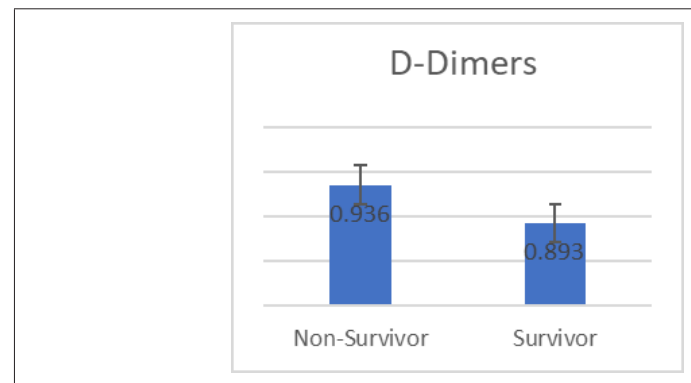

Figure: D
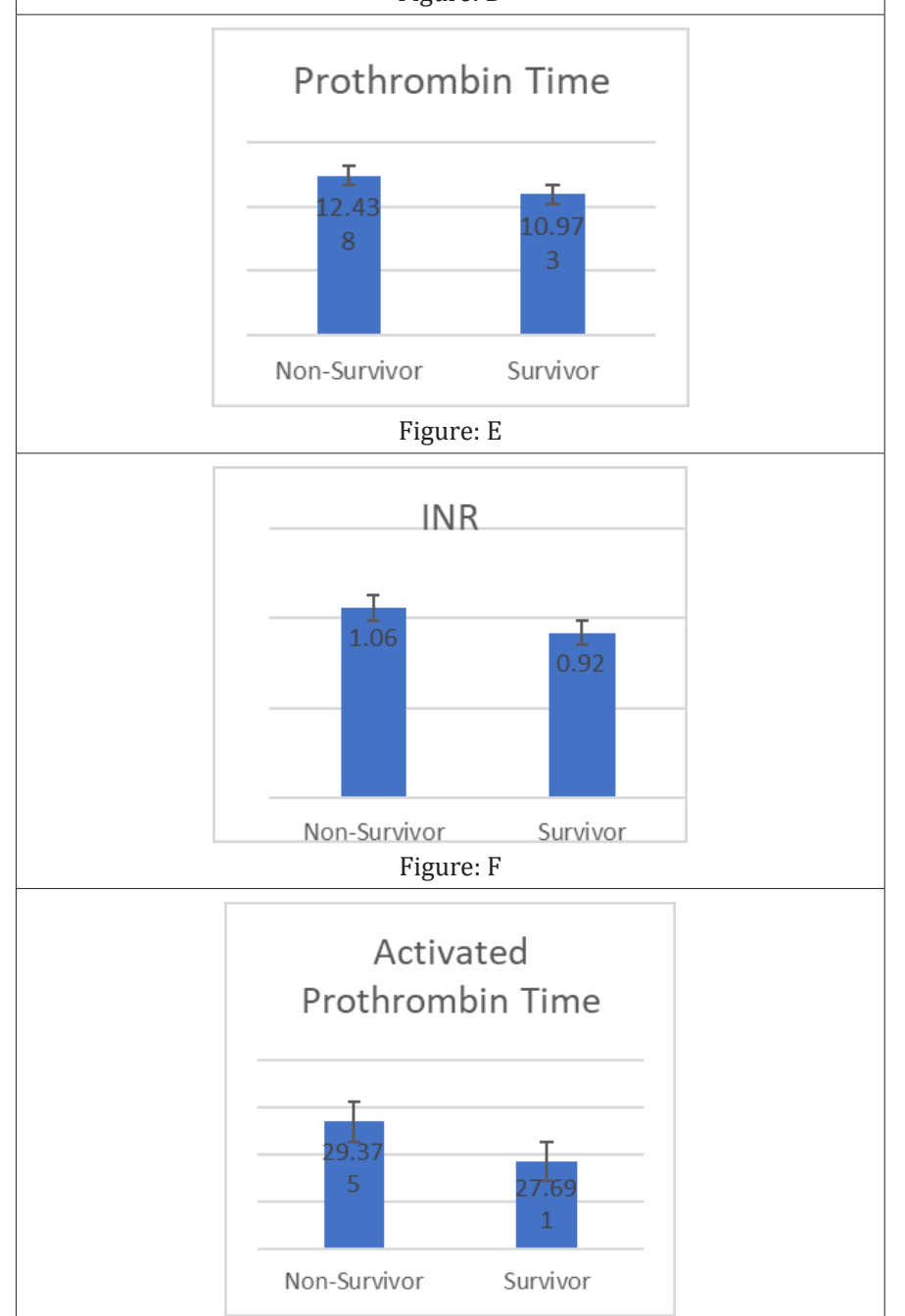

Figure: $\mathrm{G}$

Figure: A to Figure: G: Comparison of mean of Coagulation Profile in Survivors and non-Survivors.

\section{Discussion}

COVID-19 was found more prevalent in males than females in our study, in addition a greater number of males were in the non-survivor group than females. A study by Ajay Pradhan et al. conducted in September 2020 inferred that COVID-19 is not only more common in males but also shows sex biased mortality which is more in men than women [9]. Jian Min Jin in his case series study had inferred 2.4 folds increased mortality in males than females [10]. Mean Platelet count was found to be lower in the non-survivor group than the survivor-group although both groups showed mean platelet count within normal range. However, no association was found between platelet count and mortality. A study conducted by Geoffery D Wool et al. also determined that platelet count is not an indicator of mortality in COVID-19 patients [8]. A pooled SMD (standard mean difference) by Shi-Qin Jiang reported thrombocytopenia in non-survivors. Decreased Platelet counts are also seen in association of disease severity of COVID-19 [11]. Coagulopathy induced by COVID-19 disease is still a question as hemostatic dysfunctions are associated with other infections as well. A large diversity of coagulation disorders is seen in association of COVID-19.

Thrombotic Thrombocytopenic Purpura (TTP) was also seen in a patient of post- COVID-19 infection during our study which was refractory to eleven sessions of Plasma exchange and led to the mortality of the patient. Although there are many case reports on complete remission of post COVID-19, TTP patient after plasma infusions and exchanges. Fehmi Hindlerden et al. have reported a case of TTP who responded well to plasma exchange and was completely recovered after eleven sessions [12]. The values of PT and INR between survivor and non-survivor groups showed no statistical difference and no association of PT and INR was found with severity or mortality. The same was concluded by Argirios E Tasantis et al. that there is no significant difference between PT values of mild and severe disease [10]. Although many studies have established association of PT with mortality like Xin Jin et al. illustrated a strong association and prolonged PT in the nonsurvivors [16], but it was not the same in our study population. A study by Q Chen et al. has showed a strong relationship of prolonged APTT with deterioration of COVID-19 disease and prolonged APTT was seen in severe and critical cases. The same is observed in our study and APTT is seen prolonged in both survivor and non-survivor groups of COVID-19 patients but a more significant association is seen in the non-survivor group [18].

In a study conducted in Bangladesh by Bhuiyan MN concluded that $40.9 \%$ vs $16.6 \%$ showed prolonged APTT values in ICU and non-ICU patients' respectively [13]. Raised D-dimers is one of the most consistent abnormal hemostatic laboratory markers in COVID-19 patients. The association of raised D-Dimers and its link with more ICU admissions and mortality has been documented by many studies, like a study done by Maryam Aboughdir concluded that raised D-dimers were observed in ICU patients as well as inhouse deaths [14].This association was also enforced by our study where D-dimers were found raised in a lot of patients in both survivor and non-survivor group, but significantly higher levels were seen in the non-survivor group. Litao Zhang et al. has reported increased incidence of mortality in patients with D-Dimer $>0.2 \mathrm{ug} /$ $\mathrm{ml}[15-18]$. 


\section{Conclusion}

Coagulation profile in COVID-19 holds not only a prognostic value but also helps in deciding the therapeutic approach for the clinicians. The extent of coagulation dysfunctions also seems to be related with the severity of organ impairment. Coagulation abnormalities have a great impact on disease progression, severity, and prognosis. Prolonged APTT and raised D-Dimers are strongly associated with disease severity as well as poor prognosis leading to mortality of COVID-19 patients.

\section{Declarations}

Funding: The authors did not receive support from any organization for the submitted work

Conflicts of interest/Competing interests: The authors have no conflicts of interest to declare that are relevant to the content of this article.

Availability of data and material: All the data and material are available.

\section{Authors' contributions}

Conceptualization: Ambreen Kashif \& Sadia Taj; Methodology: Saira Moin; Formal analysis and investigation: Ambreen Kashif, Syeda Arzinda Fatima; Writing-original Draft preparation: Ayaz Lone; Writing-review and editing: Sadia Taj; Supervision: Qasim Ahmed

Ethics approval: The questionnaire and methodology for this study was approved by the Institutional review board of FMH college of medicine \& dentistry (IRB \#: FMH-08-2020-IRB-776-M)

Consent to Participate: Informed consent was obtained from all individual participants included in the study.

Consent to Publish: The authors affirm that participants provided informed consent for publication of the work.

\section{References}

1. Iba T, Levy JH, Levi M, Thachil J (2020) Coagulopathy in COVID-19. J Thromb Haemost 18(9): 2103-2109.

2. Shah A, Donovan K, McHugh A, Pandey M, Aaron L, et al. (2020) Thrombotic and haemorrhagic complications in critically ill patients with COVID-19: a multicenter observational study. Crit Care 24(1): 561.
3. Savioli F, Rocha LL (2020) Coagulation profile in severe COVID-19 patients: what do we know so far? Rev Bras Ter Intensiva 32(2): 197199.

4. Blasi A, Von Meijenfeldt FA, Adelmeijer J, Calvo A, Ibañez C, et al. (2020) In vitro hypercoagulability and ongoing in vivo activation of coagulation and fibrinolysis in COVID-19 patients on anticoagulation. J Thromb Haemost 18(10): 2646-2653.

5. Abou-Ismail MY, Diamond A, Kapoor S, Arafah Y, Nayak L (2020) The hypercoagulable state in COVID-19: Incidence, pathophysiology, and management. Thromb res 194: 101-115.

6. Kander T (2020) Coagulation disorder in COVID-19. Lancet Haematol $7(9): 630-632$.

7. Ranucci M, Ballotta A, Di Dedda U, Bayshnikova E, Dei Poli M, et al. (2020) The procoagulant pattern of patients with COVID-19 acute respiratory distress syndrome. J Thromb Haemost 18(7): 1747-1751.

8. Wool GD, Miller JL (2021) The impact of COVID-19 disease on platelets and coagulation. Pathobiology 88(1): 15-27.

9. Pradhan A, Olsson PE (2020) Sex differences in severity and mortality from COVID-19: are males more vulnerable? Biology of sex Differences 11(1): 53.

10. Jin JM, Bai P, He W, Wu F, Liu XF, et al. (2020) Gender differences in patients with COVID-19: focus on severity and mortality. Frontiers public health 8: 152 .

11.Jiang SQ Huang QF, Xie WM, Lv C, Quan XQ (2020) The association between severe COVID-19 and low platelet count: evidence from 31 observational studies involving 7613 participants. Br J Haematol 190(1): 29-33.

12. Hindilerden F, Yonal-Hindilerden I, Akar E, Kart-Yasar K (2020) Covid-19 associated autoimmune thrombotic thrombocytopenic purpura: report of a case. Thrombosis Research 1(195): 136-138.

13. Bhuiyan MN, Giti S, Hossen MS, Rahman MM, Zannat MN, et al. (2020) Haematologic Profile Abnormalities and Coagulopathy Associated with Covid-19: A Prospective Study of 100 Patients. J Bangladesh College Physici Surgeons 3: 61-66.

14. Aboughdir M, Kirwin T, Abdul Khader A, Wang B (2020) Prognostic value of cardiovascular biomarkers in COVID-19: a review. Viruses 12(5): 527

15. Zhang L, Yan X, Fan Q, Liu H, Liu X, et al. (2020) D-dimer levels on admission to predict in-hospital mortality in patients with Covid-19. J Thromb Haemost 18(6): 1324-1329.

16. Chen Q, Zheng Z, Zhang C, Zhang X, Wu H, et al. (2020) Clinical characteristics of 145 patients with corona virus disease 2019 (COVID-19) in Taizhou, Zhejiang, China. Infection 48(4): 543-551.

17. Tsantes AE, Frantzeskaki F, Tsantes AG, Rapti E, Rizos M, et al. (2020) The haemostatic profile in critically ill COVID-19 patients receiving therapeutic anticoagulant therapy: An observational study. Medicine 99(47): 23365.

18. Jin X, Duan Y, Bao T, Gu J, Chen Y, et al. (2020) The values of coagulation function in COVID-19 patients. Plos one 15(10): e0241329. 